\title{
Predicting Pavement Performance Utilizing Artificial Neural Network (ANN)
}

\author{
Fawaz Alharbi ${ }^{1}$, Omar Smadi²
}

\author{
${ }^{1}$ Department of Civil Engineering, College of Engineering, Qassim University;P.O. Box 6677, Buraydah, 51452, Saudi Arabia \\ Email: f.a@qec.edu.sa. \\ ${ }^{2}$ Director; Center for Transportation Research and Education, Institute for Transportation, Iowa State University; 2711 South Loop Drive, \\ Suite 4700, Ames, Iowa \\ Email :smadi@iastate.edu.
}

\begin{abstract}
Pavement management systems (PMS) play a significant role in cost-effective management of highway networks to optimize pavement performance over predicted service life of the pavements. Although, the Iowa DOT manages three primary highway systems (i.e., Interstate, US, and Iowa highways) that represent $8 \%$ (approximately 9,000 miles) of the total roadway system in the state (114,000 miles), these systems carry around $62 \%$ of the total vehicle miles traveled (VMT) and $92 \%$ of the total large truck VMT. In this research, historical climate data was acquired from relevant agencies and integrated with pavement condition data to include all related variables in prediction modeling. An artificial neural network (ANN) model was used to predict the performance of ride, cracking, rutting, and faulting indices on different pavement types. The goodness offit of the ANN prediction models was compared with the conventional multiple linear regression (MLR) models. The results show that ANN models are more accurate in predicting future conditions than MLR models. The contribution of input variables in prediction models were also estimated. The results indicated that climate factors directly influenced the pavement conditions, and ANN model results can be used by the decision makers to establish appropriate management actions to withstand harsh weather over the years.
\end{abstract}

Keywords- Pavements, Pavement management system, Artificial Neural Networks, Asphalt concrete pavements, Pavement prediction modeling.

\section{INTRODUCTION}

State highway agencies spend millions of dollars each year on maintenance and rehabilitation to meet legislative, agency, and public requirements. Effective pavement management requires a systematic approach that includes project planning, design, construction, maintenance, and rehabilitation. Pavement management systems (PMS) play a significant role in managing the condition of highway networks efficiently based on costeffective strategies to be applied at a given time for maintaining that pavement condition at an acceptable level so the pavement can satisfy the demands of traffic and environment over its service life [1]. Individual PMSs operate at two administrative levels, the network and project management levels. At the network management level, a PMS predicts the overall pavement performance for establishing budget allocations and treatment strategies. At the project management level, more detailed information and specific treatment options are required to determine when a particular pavement section may need maintenance or rehabilitation action [2].

Traffic loading and environmental factors result in pavement distress [3]. The ability to trace that distress over time allows researchers and agency decision makers to develop performance prediction models. Predicting pavement performance requires historical data about pavement conditions, traffic loading, structural characteristics, and climate data [4]. These data can be acquired from a single test road or from in-service pavements to obtain data for more practical prediction models. However, constructing and monitoring single test roads is expensive and unrealistic for small and local agencies. Developing accurate prediction models for pavements allows transportation agencies to effectively manage their highways in terms of budget allocation and scheduling maintenance and rehabilitation activities.

In this research, historical traffic loading and pavement condition data was obtained from the Iowa Department of Transportation (DOT), and climate data was acquired from the Iowa Environmental Mesonet (IEM) in order to include all related variables in the prediction models. The results of this research will improve pavement management strategies by predicting an accurate pavement distress, and evaluate the impact of Iowa weather conditions on predicting pavement performance. 
The Iowa DOT manages three primary highway systems (i.e., Interstate, US, and Iowa highways) that represent $8 \%$ (approximately 9,000 miles) of the total roadway system in the state $(114,000$ miles $)$, but these systems carry around $62 \%$ of the total vehicle miles traveled (VMT) and $92 \%$ of the total large truck VMT [5]. These highways play a major role in Iowa's economy by connecting customers and supplies across the United States, and the roads are important to economic development in several sectors such as agriculture, manufacturing, and industry. According to the Bureau of Transportation Statistics, in 2012 around 263.36 billion tons of goods valued at $\$ 195.99$ billion were transported on Iowa highways to other states [6].

Iowa faces critical challenges in providing a safe, efficient highway system, particularly in terms of balancing optimum highway conditions and available local, state, and federal funds. According to the American Society of Civil Engineers, 45\% of major roads in Iowa were in fair condition, and large truck VMT, which directly affects pavement deterioration, will increase by $66 \%$ between 2015 and 2040 [5]. The Iowa DOT has allocated about $\$ 2.7$ billion for highway construction and $\$ 1.2$ billion for improving highway safety during fiscal years 2015 to 2019 [5]. Despite these allocations, Iowa DOT administration, maintenance and construction costs result in an estimated annual funding shortfall of over $\$ 215$ million [7]

Current PMS models used by the Iowa DOT do not consider climate factors and as such, may not be accurate. Therefore, the Iowa DOT could benefit from better prediction of the future needs of pavements to ensure they serve as effectively as possible. Therefore, efforts have been made to acquire historical climate data from Iowa Environmental Mesonet (IEM) and integrate it with pavement condition data from Iowa DOT. Then pavement performance models could be developed for asphalt, concrete, and composite pavements.

In addition to pavement performance modeling, some transportation agencies (e.g., cities and counties) do not have the capability for conducting deflection tests on their roadways for structural evaluation because these tests generally require specialized equipment, experience, and knowledge. As a result, these agencies may rely primarily on functional condition data to assess the strength of their roadways. However artificial neural network (ANN) pavement prediction models have been developed that can account for both the pavement conditions and the climate data and can estimate the relationship between structural capacity and rutting depth in asphalt pavement at the network level.
This research aims to explore pavement performance prediction models that can help decision makers take appropriate actions by developing and comparing traditional regression and artificial neural network models in predicting future pavement conditions for asphalt, concrete, and composite pavements and support pavement management decisions, and ii) determining the impact of various input variables on the deterioration of pavement conditions.

\section{METHODS}

Predicting pavement performance is often considered to be a difficult task because of several influencing factors. Accurate pavement performance models, including large data, are needed to develop pavement maintenance and rehabilitation strategies. Varying causes of pavement deterioration from one road section to the next makes the modeling of pavement performance a complex process. In this research, multiple linear regression (MLR) and artificial neural network (ANN) models were used to predict pavement performance. Also, the results of ANN models are analyzed to determine the relative contribution of each variable on several distress indices.

Pavement distress data was obtained from the Iowa DOT Pavement Management Information System (PMIS) and climate data was obtained from the Iowa Environmental Mesonet (IEM). Iowa highways are classified into three systems: Interstate highways, US highways, and Iowa highways. The most recent PMIS data is presented in Figure 1. It can be seen in the Figure that Iowa highways system has the longest highways in Iowa, and $55 \%$ of these highways are composite pavements.

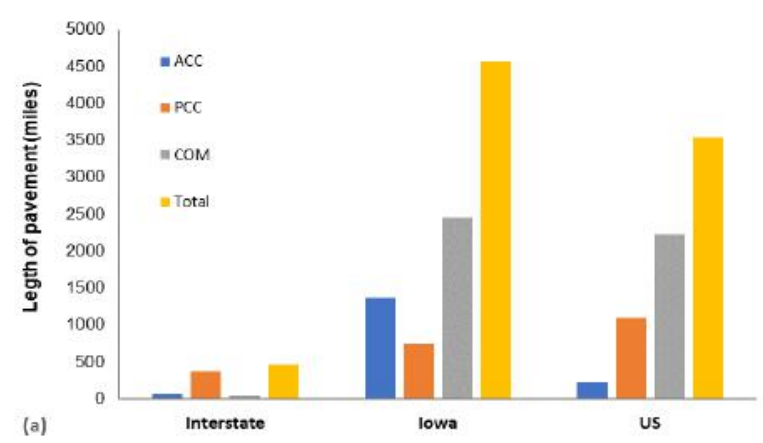

Fig. 1: Length of Iowa highway systems with different types of pavement 
Geographic information system (GIS) software was used to integrate the PMIS road condition data and the IEM climate data for each location under study. The GIS spatial integration process provided accurate overlays of the weather stations over Iowa highways. The GIS was also used to display weather conditions as colored maps. To ensure that the climate data and locations of each pavement section were associated, each pavement section was assigned to the closest weather station. For example, Figure 2 shows the 32 pavement sections that were closest to weather station IA0133.

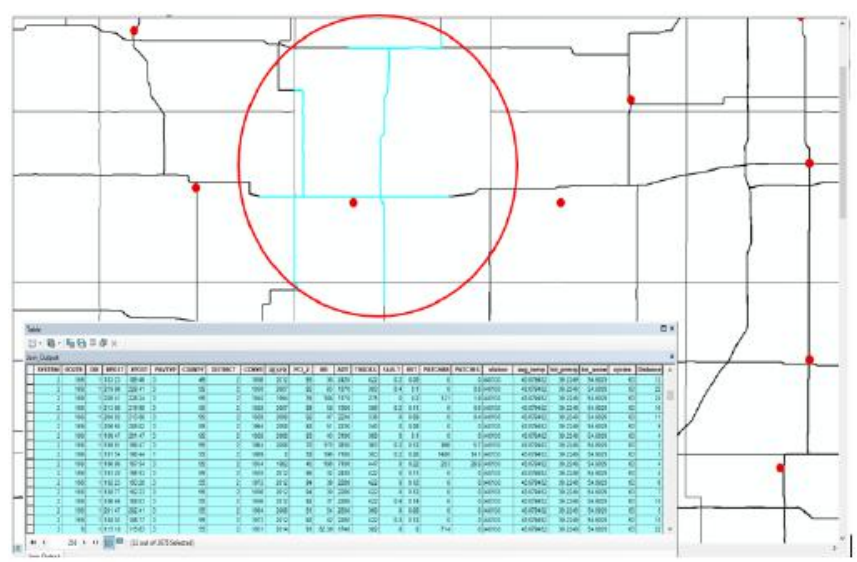

Fig. 2. Pavement sections associated with the closest weather station

Two kinds of pavement performance models, traditional multiple linear regression (MLR) and artificial neural network (ANN) models are developed to study three pavement types with different pavement properties and different material properties. Goodness of fit is a common measure for evaluating model performance. The coefficient of determination $\left(\mathrm{R}^{2}\right)$ and root mean square error (RMSE) were utilized to measure and compare the performance of the models.

Historical data was used on in both ANN and MLR models to predict individual distresses for three pavement types, ACC, PCC, and COM pavements. These individual distresses were predicted based on weather factors (i.e., temperature, precipitation, and freeze-thaw cycles), traffic loading, pavement age, structure number, layer thicknesses, and subgrade stiffness. For ACC and COM pavements, three models were developed for predicting roughness, cracking, and rutting, and for PCC pavements, three models were developed for predicting roughness, cracking, and faulting. These predicted distresses were aggregated to calculate the PCI values based on Equations 1 and 2 in order to represent the overall PCI over the years. To determine the reliability of ANN models, the results obtained from the ANN models were compared with the results from MLR models.
PCI $_{\text {Com\&ACC }}=0.4($ Cracking Index $)+0.4($ Riding Index $)+$ 0.2 (Rutting Index)

$\mathrm{PCI}_{\mathrm{PCC}}=0.4($ Cracking Index $)+0.4($ Riding Index $)+$ 0.2 (Faulting Index)

Three main components are used to develop ANN models: i) the structure of connection between input and output layers (architecture); ii) the method of adjusting the connection weight (learning method); and iii) the neuron activation function.

There is no standard method for selecting the appropriate number of neurons, so training the ANN model with sequential number of hidden neurons and then selecting the number of neurons that achieve minimum RMSE was employed. The lowest RMSE value was achieved at 12 hidden neurons for predicting cracking index in COM pavement. The final layer in the structure of ANN model is the output layer that produces the result from processing provided by the hidden layer.

After determining the architecture of an ANN model, software (e.g., JMP) is used to randomly divide the database. Seventy percent $(70 \%)$ of the database was used for training and thirty percent for validation of ANN model. The training data set is used to develop the model, while the validation data is used to assess the accuracy of the ANN model and avoid overfitting in the model [8]. The results of the training process produce the weight matrices that are stored in links between layers and that can also be used to extract information about the contribution of each input in the model output. In the training process, the connection weights between the layers is adjusted, thereby, minimize the overall mean error by using back-propagation algorithm. The basic unit of an ANN model is a neuron that combines inputs and produces an output as shown in Figure 9. The neurons are not individually powerful in terms of computational power, but their interconnection with neurons in different layers produces the desired relationship among variables and demonstrates the processing capabilities of the neurons [9].

Multiple linear regression (MLR) models were used to predict values of pavement conditions (ride, cracking, rutting, and faulting) and those results were compared with prediction results from ANN modeling. MLR modeling has been widely used by highway agencies to predict pavement performance and deterioration rates because of its simplicity and ease of implementation [10].

\section{RESULTS AND DISCUSSION}

The analysis showed that the ANN models yield 
reasonably accurate models compared to the results of MLR models.

For ACC pavements, the ANN models yielded more accurate predictions than the MLR models in riding, cracking, and rutting indices. The $\mathrm{R}^{2}$ values indicate there are good correlations between actual and predicted indices for each index. The $\mathrm{R}^{2}$ values from the ANN models are higher than the $\mathrm{R}^{2}$ values of the MLR models by $61.40 \%, 48.15 \%$, and $48.15 \%$ for riding, cracking, and rutting index, respectively. For PCC pavements, the ANN models achieve better accuracy models with improvement in $\mathrm{R}^{2}$ by $23.00 \%, 26.93 \%$, and $80.00 \%$ than MLR models in predicting riding, crack, and fault indices, respectively, compared with MLR models. For COM pavements, the comparison of the results produced by ANN and MLR models showed that the ANN model performed better accurate models than MLR models.

After predicting the pavement condition indices, the weights and biases matrices from ANN layers were used to predict the future performance of riding, cracking, rutting, and faulting indices assuming no future treatment will be applied. The adjusted weights were developed by a back-propagation algorithm during the training process in order to make the predicted values close to the actual values. The weights and biases matrices were stored by JMP software. Predicting the future pavement conditions was predicted incrementally; for instance, the first predicted value for pavement condition in age $(t+1)$ was used to predict the second year pavement condition $(t+2)$ as the input of the previous pavement condition and so on. three pavement sections, one each of ACC, PCC, and $\mathrm{COM}$ pavements, were selected to analyze their performance over the years and predict future performance. The curves for each pavement section begin at the year of construction, and it is assumed that no maintenance has been or will be done on the section.

The performance curve for an ACC pavement section from I-35 is shown in Figure 3. The performance curve shows that the pavement section was in poor condition, (i.e., the PCI value is lower than 41) 11 years after construction.

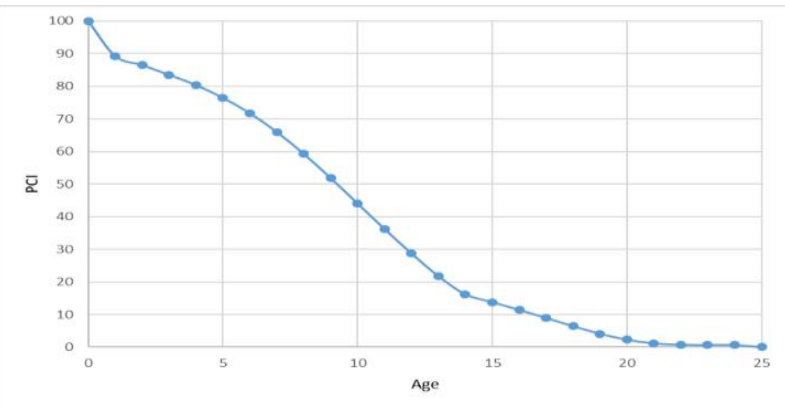

Fig. 3. Pavement performance curves of I-35 section (ACC pavement)

Figure 4 shows the performance curve of a pavement section of US-30 in Story County. The pavement type of this section is a composite pavement with PCC overlaid by ACC pavement. Composite pavement is widely used in Iowa, with around $46 \%$ of the total highway. The composite pavements typically last longer than ACC pavement because the concrete base layer makes it a stronger structure. The US-30 pavement section can serve in good or fair condition up to approximately 20 years, which is better performance than the ACC pavement section.

Figure 5 shows the performance curve of a PCC pavement section of Iowa highway 1 . This performance curve indicates that PCC pavement lasts longer than both ACC and COM pavements and that PCC deteriorates at a lower rate early age. The curve indicates that this pavement section should be in good or fair condition for almost 40 years.

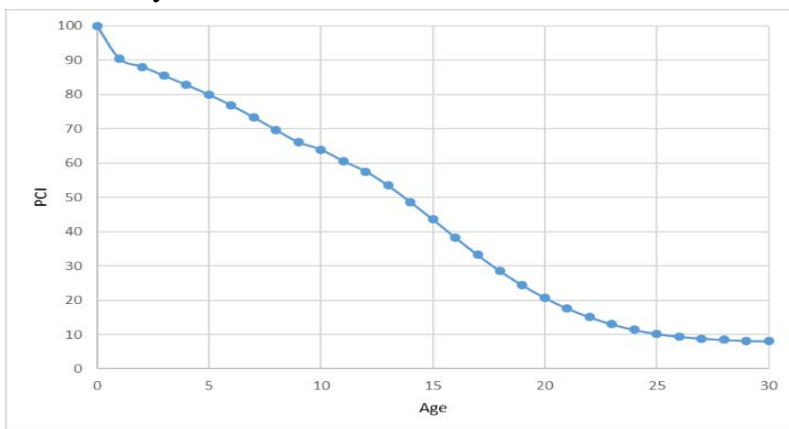

Fig. 4. Pavement performance curves of US-30 section (COM pavement)

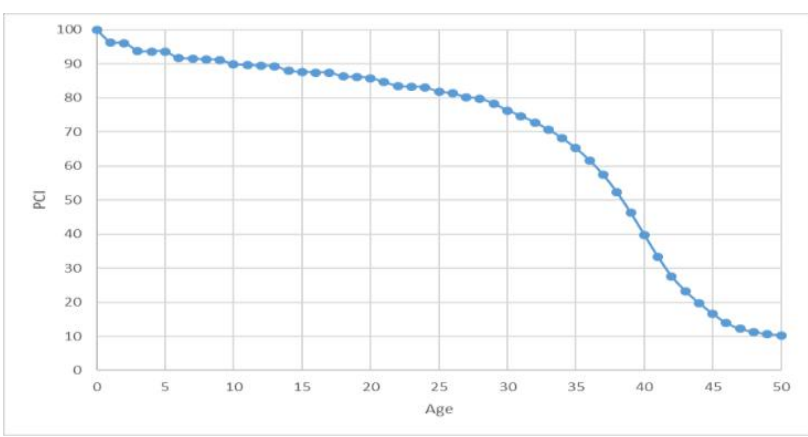

Fig. 5. Pavement performance curves of Iowa-1 section (PCC pavement)

\section{CONCLUSION}

Predicting future pavement performance is essential for allocating available funding for maintenance and rehabilitation activities at the network level as well as for determining the most cost-effective strategies at the project management level. The models in this study were 
developed to include climate data in addition to historical pavement condition data. Results of the ANN models were found to be more accurate than the MLR models, perhaps because ANN models can deal with larger, more complex data sets.

\section{REFERENCES}

[1] Bianchini, A. and Bandini, P., 2010. Prediction of pavement performance through neuro-fuzzy reasoning. Computer- Aided Civil and Infrastructure Engineering, 25 (1), 39-54. doi:10.1111/j.1467-8667.2009.00615.x.

[2] Geoffroy, D.N., Shufon., J.J., (1992) Network-Level Pavement Management in New York State: A GoalOriented Approach. Transportation Research Record 1344 (1992).

[3] Adlinge, S. S., Gupta, A. K. (2013). Pavement deterioration and its causes. International Journal of Innovative Research and Development, 2(4), 437-450.

[4] Ziari, H., Sobhani, J., Ayoubinejad, J., Hartmann, T. (2016). Prediction of IRI in short and long terms for flexible pavements: ANN and GMDH methods. International Journal of Pavement Engineering, 17(9), 776-788.

[5] ASCE. (2015). Iowa infrastructure 2015 report card. American Society of Civil Engineers.

[6] BTS. (2012). U.S. Department of Transportation. 2012 Commodity Flow Survey, State Summaries. Bureau of Transportation Statistics (BTS).

[7] Iowa DOT. (2016). 2016 road use tax fund (RUTF) study. Iowa Department of Transportation.

[8] Ling, M., X. Luo, S. Hu, F. Gu, \& R. L. Lytton. (2017). Numerical modeling and artificial neural network for predicting $\mathrm{J}$-integral of top-down cracking in asphalt concrete pavement. In Transportation Research Board 96th Annual Meeting (No. 17-05318).

[9] Attoh-Okine, N. (1994). Predicting roughness progression in flexible pavements using artificial neural networks. Transportation Research Board Conference Proceedings, (Vol. 1, No. 1).

[10] Xu, G., L Bai, Z. Sun, T. Nowaczyk, C. Shive, \& J.Wilcoxson. (2015, May). Pavement Deterioration Prediction Model and Project Selection for Kentucky Highways. In 9th International Conference on Managing Pavement Assets. 